\title{
L'évaluation en langues à l'entrée de la fonction publique en France et au Canada
}

\section{Anne-Marie Barrault-Méthy}

\section{OpenEdition}

\section{Journals}

Édition électronique

URL : http://journals.openedition.org/asp/421

DOI : $10.4000 /$ asp. 421

ISBN : 978-2-8218-0406-7

ISSN : 2108-6354

Éditeur

Groupe d'étude et de recherche en anglais de spécialité

Édition imprimée

Date de publication : 1 novembre 2008

Pagination : 159-183

ISSN : 1246-8185

Référence électronique

Anne-Marie Barrault-Méthy, « L'évaluation en langues à l'entrée de la fonction publique en France et au Canada », ASp [En ligne], 53-54 | 2008, mis en ligne le 01 novembre 2011, consulté le 01 mai 2019.

URL : http://journals.openedition.org/asp/421 ; DOI : 10.4000/asp.421

Ce document a été généré automatiquement le 1 mai 2019

Tous droits réservés 


\title{
L'évaluation en langues à l'entrée de la fonction publique en France et au Canada
}

\author{
Anne-Marie Barrault-Méthy
}

1 Cette étude constitue une introduction à l'analyse des modalités d'évaluation en langue dans la fonction publique de pays anglophones ainsi que de pays membres du Conseil de l'Europe. Le présent travail s'attache à décrire des systèmes que les enseignants français préparateurs aux concours administratifs, civilisationnistes ou spécialistes de langue de spécialité, connaissent parfois peu. Ces collègues se sont immanquablement posé les questions suivantes, relatives à la didactique et à la civilisation. Questions didactiques, d'abord: quels sont les critères d'évaluation des futurs fonctionnaires? Quelle langue de spécialité enseigner à nos étudiants qui préparent les concours administratifs? Questions de civilisation ensuite: comment se passe l'évaluation en langue dans l'entrée à l'administration dans les pays anglophones? Dans quelle langue est-on évalué, par qui et selon quels critères? Les études globales sur ces questions font défaut. Pourtant, l'évaluation en langues à l'entrée de l'administration coûte cher et les enjeux sont importants dans un contexte d'augmentation des échanges internationaux, y compris dans la fonction publique, et d'évaluation des dispositifs publics, avec en toile de fond les recommandations du Conseil de l'Europe sur l'évaluation et la conception de tests.

2 Le premier pays sur lequel porte notre étude comparative est le Canada. En effet, dans ce pays officiellement bilingue, les citoyens peuvent s'adresser à leur administration indifféremment en français ou en anglais, ce qui impose une efficacité managériale ainsi qu'un haut niveau de compétence linguistique pour certains fonctionnaires. Le système d'évaluation linguistique vient d'être remanié ${ }^{1}$ après un chantier de plusieurs années. Le Canada s'est doté d'un système de niveaux analogues à ceux du Conseil de l'Europe, attribuant aux compétences une lettre en fonction du niveau de maîtrise de l'une des deux langues officielles, la lettre A indiquant un niveau faible, B, un niveau intermédiaire et $\mathrm{C}$, un niveau élevé. Nous décrivons ici le système français d'évaluation à l'entrée de l'administration et le système canadien d'ELS (évaluation de la langue seconde) et les 
comparons au Cadre européen commun de référence (CECR) pour finalement formuler des propositions visant à faire évoluer l'évaluation dans les concours administratifs français.

\section{Pourquoi évaluer les langues aux concours?}

3 Les épreuves de langues remplissent des fonctions très différentes dans les deux pays. Au Canada, les postes exigeant des compétences dans les deux langues se voient affecter un niveau dans la langue seconde. Par exemple, pour un poste de directeur général / directrice générale, service fédéral de médiation et de conciliation, poste pour lequel le bilinguisme est requis, la Commission de la fonction publique demande un niveau minimum identique en français et en anglais: $C$ en compréhension de l'écrit, $B$ en expression écrite et $\mathrm{C}$ en interaction orale. Les compétences sont donc directement mises en œuvre par le fonctionnaire.

4 En France, les administrations ne recrutent pas pour un poste, mais pour un corps. Il existe quelques exceptions, comme les concours d'officiers de port ou de contrôleurs aériens ainsi que la fonction publique territoriale. Le principe général est de proposer une affectation après le concours, éventuellement après un passage dans une école de formation, souvent en fonction d'un classement intégrant les performances globales des nouveaux recrutés dans un certain nombre de matières. Les langues font partie des connaissances globales évaluées par le concours, au même titre que la culture générale, le droit administratif, l'économie ou la capacité à rédiger une note de synthèse ou parfois le sport. Les écoles de formation prévoient parfois une formation en langue mais pas dans toutes les langues offertes aux concours (il s'agit le plus souvent d'une formation en anglais). Dans la fonction publique d'État, les besoins en langues dans les postes à pourvoir ne sont, le plus souvent, pas évalués préalablement.

\section{Les langues dans les concours administratifs français}

5 La France ne possède qu'une langue officielle mais vise officiellement pour ses citoyens le multilinguisme pour favoriser la mobilité de la main d'œuvre.

6 La présente étude s'appuie sur l'analyse des 536 concours de la fonction publique d'État et de la fonction publique territoriale que nous avons recensés. Notre travail ne couvre donc pas les autres fonctions publiques, à savoir les fonctions publiques hospitalières, la fonction publique de la ville de Paris et la fonction publique européenne. Les données ont été recueillies entre 2000 et 2006. Le dénombrement des concours est une opération extrêmement difficile. Les concours sont nombreux, organisés par des administrations très diverses : ministères, juridictions (comme le Conseil d'État), Hauts Commissariats de la République, secrétariats d'État, services du Premier ministre. Certains ministères soustraitent la gestion de concours à d'autres administrations. Le nombre de fonctionnaires à un moment donné n'est pas connu avec précision. La fonction publique représentait à peu près 5,2 millions de personnes en France au 31 décembre 2003, selon le rapport annuel 2004-2005 de l'Observatoire de l'emploi public, Nous pensons que les concours que nous analysons dans cette étude représentent une grande majorité des postes ouverts au 
recrutement et qui figurent dans la base de données de la direction générale de l'administration et de la fonction publique.

7 Nous avons exclu de l'étude les concours d'enseignement qui font l'objet d'un rapport de jury détaillé tous les ans. Nous avons aussi exclu les concours d'accès aux Grandes Écoles (type École Normale Supérieure) lorsqu'elles ne donnent pas accès au statut de fonctionnaire. Nous avons collecté les données en contactant par téléphone les services des examens et concours des administrations, en visitant leur site et en rassemblant les sujets et les rapports de jury chaque fois qu'ils étaient disponibles, en consultant le Journal Officiel, en interrogeant les étudiants qui suivent ou ont suivi une préparation spécifique à l'Institut de préparation à l'administration générale de Poitiers, le tout pendant dix ans (1997-2007). Nous avons également rencontré deux présidents de jury et plusieurs examinateurs et nous avons assisté à des épreuves de concours ${ }^{2}$ dans deux jurys différents. Certaines épreuves n'ont pas été répertoriées, soit parce que le concours n'était pas ouvert au moment de l'enquête, soit que les renseignements disponibles n'aient pas été complets. ${ }^{3}$ Les données ont été traitées à l'aide du logiciel Access. ${ }^{4}$ Au delà de la diversité des épreuves, l'évaluation des compétences en langues aux concours administratifs français semblent constituer un système. Nous nous proposons de dresser une typologie des épreuves puis d'évaluer la validité de l'évaluation.

\subsection{Typologie des épreuves 5}

8 L'accès à un corps peut faire l'objet de plusieurs concours différents (interne, externe, « 3 e concours", destiné aux candidats ayant acquis une expérience dans l'exercice d'une activité professionnelle, d'un mandat d'élu local ou d'une activité associative, concours professionnels, concours déconcentrés, etc.) dont les épreuves peuvent être différentes, de sorte qu'un concours interne peut ne pas comporter d'épreuve de langue alors qu'il en existe au concours externe. ${ }^{6}$ Certains concours ne comportent pas d'épreuve de langue alors que la maîtrise d'une langue, en général l'anglais, est importante dans le poste. ${ }^{7}$

Les langues ne sont pas traitées à égalité. L'anglais est parfois obligatoire, comme dans les concours des ministères des affaires étrangères ou la direction générale de l'aviation civile. Le plus souvent cependant, les administrations offrent aux candidats un choix entre l'anglais, l'allemand et l'espagnol, avec parfois le russe, l'italien, l'arabe et même le chinois mandarin. Le concours dont le choix de langues est le plus vaste est celui de l'ENA: pas moins de quatorze langues sont proposées. Concernant les langues régionales, le seul concours qui semble offrir des épreuves de ce type est celui de conseiller d'éducation populaire et de la jeunesse, qui propose un choix de sept langues régionales en plus des langues étrangères, mais il reste l'exception; à noter que le baccalauréat offre, lui, un choix de langues étrangères et régionales beaucoup plus vaste. ${ }^{8}$ En outre, aucun concours ne comporte d'épreuve de langue régionale étrangère. Dans l'ensemble, le panel de langues semble relever du choix politique de l'administration organisatrice.

137 concours sur 536, soit 25,5\%, comportent au moins une épreuve de langue. Nous avons recensé 25 types d'épreuves au total (cf. tableau en annexe 1). Nous disposons d'informations sur la nature de l'épreuve de langue pour 124 concours. En dépit de l'hétérogénéité des épreuves, on remarque une homogénéité globale de l'esprit des exercices. À l'écrit, l'épreuve reine est la version (27\% des épreuves de langues), le plus souvent sans dictionnaire. La majorité des épreuves de langues sont orales (19 types d'épreuves sur 26), et il s'agit alors d'une version assortie éventuellement d'une 
conversation, d'une conversation libre ou du trio lecture / version / conversation. La version, à l'écrit ou à l'oral, apparaît au total dans 59,1\% des épreuves de concours analysées ici.

11 La plus grande partie des concours $(90,5 \%)$ se contentent d'une seule épreuve, soit écrite, soit orale. Les concours des ministères des affaires étrangères et de la direction générale de l'aviation civile sont ceux qui testent les candidats de façon plus complète.

\subsection{La validité}

12 La validité est le fait, pour une évaluation, de mesurer de façon réelle, précise et cohérente ce qu'elle est censée mesurer. Il existe plusieurs sortes de validité, que nous examinerons tour à tour.

\subsubsection{Validité du contenu}

Le contenu des épreuves de langue peut être considéré comme valide si est évalué un échantillon représentatif des compétences, des structures, du vocabulaire, etc., si les items sont en rapport avec le niveau attendu (Hughes 2003: 36), si les candidats sont interrogés sur ce que doivent connaître les fonctionnaires. Dans une logique de corps de fonctionnaires polyvalents, les ministères et administrations organisatrices ne font pas état d'une analyse des besoins linguistiques langue par langue, par corps et par affectation des futurs fonctionnaires. Les administrations se bornent à définir des compétences générales que devraient posséder les lauréats, par exemple la capacité à comprendre un article de presse et à en restituer le contenu oralement. Ces derniers peuvent ne jamais avoir ensuite à se servir de la langue dans leurs attributions. Cela explique peut-être la présence de certaines langues modimes (moins diffusées, moins enseignées), comme le néerlandais et le grec, à des concours comme attaché territorial. Ensuite, le niveau de maîtrise de la langue requis par concours n'est en général pas défini avec précision. Le concours d'officier de port est le seul qui renvoie les candidats à un référentiel. ${ }^{9}$

Plus généralement, les concours qui permettent d'accéder aux rapports des jurys des années précédentes restent l'exception. Entre les sujets d'anglais proposés aux concours de catégorie $A, B$ et $C$, qui requièrent des niveaux de diplômes différents, ${ }^{10}$ il n'existe pas de différence manifeste de longueur ni de niveau de difficulté ${ }^{11}$ du passage à traduire (Méthy \& Monglon 2000b).Certains sujets, trop longs, sont en fait impossibles à traiter dans le temps imparti aux candidats. C'est le cas notamment du texte à traduire en une heure pour le concours externe d'informaticien (corps des administrateurs adjoints, catégorie A) de l'Assemblée nationale, en septembre 1998, qui comptait 496 mots. Hormis l'épreuve d'anglais du concours d'officier de port, aucune épreuve de concours ne possède de validité de contenu.

\subsubsection{Validité simultanée des critères d'évaluation}

Elle est mesurée en comparant les résultats de l'évaluation à tester aux résultats d'une évaluation pour tous les items relatifs à un niveau donné (Hughes 2003 : 27), que celle-ci soit une évaluation plus longue ou bien une évaluation fiable d'un étudiant par un enseignant. 
le jury est constitué d'enseignants de langues rompus à l'évaluation du fait de leur expérience en lycée ou en université, permettent de se faire une idée de la capacité d'un candidat à s'exprimer sans préparation sur une diversité de sujets. Cependant - et ceci est vrai pour tous les concours examinés - comme il n'existe pas de référentiel des compétences, ni en fonction du niveau de langue, ni de la langue, ni du poste à pourvoir, les critères d'évaluation à l'écrit comme à l'oral n'ont aucune validité simultanée.

\subsubsection{Validité prédictive}

17 La validité prédictive d'une évaluation est sa capacité à prévoir la performance future d'un candidat lorsqu'il sera en situation dans sa vie professionnelle (Hughes $2003: 29$ ). Les épreuves de langues aux concours n'en ont aucune. En effet, il n'existe pas de description, par poste à pourvoir, des compétences en langues nécessaires.

\subsubsection{Validité théorique ${ }^{12}$}

Pour la mesurer, il faut se demander ce que doivent être les compétences en langues pour le corps pour lequel on recrute et comment les mesurer. À notre connaissance, il n'existe pas d'étude précise par corps, ni à plus forte raison par poste, permettant de définir les besoins, dans quelles langues et avec quel(s) niveau(x) de compétence. La plupart des épreuves de langues intègrent un document rédigé en langue étrangère dont il est demandé une traduction écrite ou orale ; pour les concours des bibliothèques, il s'agira d'un texte littéraire et, pour les autres concours, un article de presse, avec un contenu lexical parfois en rapport avec le corps pour lequel sélectionne le concours. Toutefois, les concours s'attachent rarement à évaluer les compétences en langue de spécialité (un savoir propre à une communauté de discours et qui relève d'un champ d'expérience de la profession), ou langue spécialisée (objet linguistique d'une communauté de discours), propre à une profession ou à l'administration en général. Dans la mesure où les candidats préparent souvent plusieurs concours, et où les langues de spécialité font rarement l'objet d'un enseignement spécifique, les administrations ne s'attendent pas à ce que les candidats les maîtrisent. Concernant la civilisation des pays où la langue est employée, elle ne fait pas directement l'objet de l'évaluation, même si des connaissances dans ces domaines sont souvent sollicitées pour la compréhension d'un texte écrit ou à l'oral pendant le dialogue avec le jury. Lorsque ce jury est composé de professionnels, on peut estimer qu'il possède une connaissance empirique des besoins en langues par corps mais pas par poste, puisque cela est déterminé bien après le concours. En revanche, on peut s'interroger sur ce que les enseignants qui siègent aux concours savent de ces besoins.

\subsubsection{Validité de la notation}

Lorsque les membres du jury sont des professionnels et non des enseignants, ils ont tendance à faire abstraction des erreurs de placement de l'accent tonique et de prononciation des voyelles dans leur conversation avec les candidats. Or, ces erreurs entravent la compréhension par des locuteurs natifs, voire la rendent impossible.

20

version, parce qu'elle évalue la langue cible (L2) à travers le français langue maternelle (L1), ne permet pas de distinguer avec certitude une maîtrise insuffisante de la L1 par rapport à celle de la L2. Un faux-sens peut conduire à un contresens et dans ce cas, le jury 
peut prendre en compte soit la source de l'erreur (l'ignorance d'un mot), soit le résultat (l'ignorance du sens d'un passage). Concernant les fautes d'orthographe, les administrations ne donnent pas systématiquement d'instructions aux jurys concernant leur pénalisation. Il en est de même pour la grammaire française, que certains candidats, étrangers ou dont le français n'est pas la langue maternelle, connaissent plus ou moins bien. Si, à l'oral, l'accent d'un candidat ou son nom révèle parfois une origine étrangère, l'écrit reste anonyme.

Par conséquent, les 59,1\% des épreuves qui comportent une version manquent de validité dans la notation. Il faut ajouter à ce chiffre tous les concours dont les membres du jury à l'oral ne sont ni des locuteurs natifs, ni des enseignants de la langue.

\subsubsection{Validité apparente}

D’une façon générale, on peut être réservé sur la validité apparente (Hughes $2003: 33)^{13}$ de l'évaluation de la compréhension de l'écrit à travers la version parce que la traduction mesure la compréhension non directement, mais par rapport à une production en français. La version, exercice issu de la méthodologie traditionnelle (Puren 1988), est héritée de l'enseignement du grec et du latin tel qu'ils étaient pratiqués dans l'ensemble de l'Enseignement secondaire du XVII ${ }^{\mathrm{e}}$ au XIX ${ }^{\mathrm{e}}$ siècle. Les enseignants qui participent à des jurys de concours administratifs évaluant les candidats, pour tout ou partie de l'épreuve, à travers des versions, s'inscrivent dans une tradition et une continuité : euxmêmes ont été soumis à cet exercice lorsqu'ils ont passé les concours d'enseignement des langues. La loi d'isomorphisme, qui veut que «le formateur tende spontanément à reproduire dans sa pratique ce qui a présidé à sa propre formation» (Puren 1988:52), s'applique ici alors que le modèle théorique de l'apprentissage qui sous-tend l'exercice de version n'a plus cours.

Dans la mesure où la plupart des concours ${ }^{14}$ n'évalue qu'une partie des compétences, et où le construct, c'est-à-dire ce qui est mesuré, n'est pas défini précisément, les concours n'ont donc pas de validité apparente.

Enfin, du point de vue stylistique, les renseignements sur les épreuves de langues aux concours administratifs en France font l'objet de tournures impersonnelles ou à la troisième personne du singulier: les administrations se contentent d'énoncer des procédures.

Les caractéristiques de l'évaluation en langue étrangère dans les concours sont :

- l'autonomie des administrations en matière d'évaluation. Il existe une décentralisation extrême des décisions. En pratique, ce sont les présidents de jury et le plus souvent l'enseignant ou le professionnel responsable qui définissent les épreuves ;

- le faible cadrage des jurys, c'est-à-dire le peu d'instructions quant aux critères d'évaluation ;

- le plus souvent, l'absence de validité de l'évaluation.

Heureusement, certaines administrations, par exemple le ministère de l'Intérieur pour le concours de lieutenant de police, effectuent un réel travail de réflexion, et parfois de communication, sur les épreuves et répondent aux besoins de terrain en langue étrangère des lauréats en aval des concours par une offre de stages de langue étrangère spécialisée.

Il n'en demeure pas moins que les concepteurs d'évaluation, même s'ils sont des enseignants expérimentés, ne sont pas épaulés par des spécialistes de psychométrie, un 
paradoxe compte tenu de l'importance de l'enjeu des concours pour les candidats et la collectivité.

\section{Les langues à l'entrée de l'administration au Canada}

L'évaluation en langues à l'entrée de la fonction publique diffère de celle pratiquée en France, ne serait-ce que parce que les langues se limitent au français et à l'anglais. La fonction publique doit pouvoir satisfaire les besoins de ses administrés en deux langues et seulement deux. La Commission de la Fonction Publique du Canada (CFP-PSC) vient de moderniser les modalités d'accès à la fonction publique après un chantier de plusieurs années. Nous nous proposons de comparer le référentiel des niveaux qui est entré en vigueur le 31 décembre 2005 avec le Cadre, puis d'examiner la validité de l'évaluation selon les critères en vigueur depuis cette date.

\subsection{Description}

Le descriptif des niveaux (CFP-PSC 2005), ventilés sous forme de tableau pour faciliter la comparaison avec le Cadre, est présenté en annexe 2. Ce référentiel ne saurait se résumer à une simplification de la grille du Conseil de l'Europe. Il résout trois questions laissées en suspens par le Cadre, à savoir l'absence de compétence, l'expertise et la place de la langue de spécialité.

Comme dans le Cadre, la perspective dans laquelle sont décrits les niveaux n'est pas culturelle, puisque la langue est le véhicule de la culture nationale, mais actionnelle : aux niveaux $\mathrm{A}, \mathrm{B}$ et $\mathrm{C}$ correspondent des tâches précises, tandis que $\mathrm{X}$ indique qu'un candidat n'est pas capable d'agir dans l'autre langue officielle. À l'écrit du niveau A, il s'agit de « [... ] parcourir des lettres, des notes de service ou des formulaires afin de les classer ou de les acheminer vers les agents responsables; parcourir divers textes afin d'en dégager des éléments d'information précis (dates, chiffres, noms, etc.); prendre connaissance de formulaires tels que des demandes, des commandes et des factures, afin de les traiter; et parcourir des listes, des index, des relevés d'ordinateur en vue de compiler des données ou de noter des changements $»{ }^{15}$ Ce sont les tâches de l'agent au contact à la fois avec le public, les fournisseurs et les supérieurs hiérarchiques. Elles supposent une compréhension partielle et littérale des documents.

Le niveau inférieur de compétence définit déjà des compétences concrètes relevant de la langue de spécialité. En interaction orale, un candidat à la fonction publique de niveau $\mathrm{A}$ est capable de « répondre à des demandes simples [...] au sujet de fournitures, de dossiers, d'équipement, de documents et de lettres ». La terminologie à connaître concerne les objets concrets du bureau. Cette terminologie du Conseil de l'Europe qui, pour les niveaux inférieurs, évoque la simplicité des tâches, se retrouve dans le référentiel de la CFP-PSC mais le contexte d'utilisation est précisé. Contrairement au Cadre (Petit 2006), le seul « versant public » est évalué : ce n'est pas le niveau de l'usager des services publics que la CFP-PSC teste, mais le niveau du seul professionnel au contact du public.

Les tâches que doit accomplir le futur fonctionnaire diffèrent selon le niveau de compétence. Au niveau A, il s'agira essentiellement de tâches d'exécution, tandis que le niveau $B$ définit des tâches de coordination avec par exemple des collègues de l'autre langue officielle. Au niveau B, il faut également être capable de hiérarchiser les 
informations, de les analyser, de les interpréter, de les vérifier, de les superviser et de les contrôler. Le niveau $\mathrm{C}$ implique davantage de responsabilités. En compréhension écrite par exemple, le fonctionnaire devra «évaluer les répercussions, [...] formuler des commentaires ou [...] faire des recommandations»; en interaction orale, il devra « expliquer des politiques, procédures, règlements, programmes et services reliés à un domaine de travail et en discuter; participer efficacement à des discussions qui comportent un échange rapide d'idées ». Les tâches de niveau C correspondent à des postes d'encadrement, des postes sensibles, où les fonctionnaires analyseront des énoncés complexes et abstraits et où leur capacité d'abstraction sera sollicitée en L2. Ils doivent pouvoir participer à une décision politique ou administrative, exercer leur jugement, expliquer, synthétiser et présenter leur travail. Les tâches à accomplir, pour tous les niveaux, sont définies clairement.

Une autre différence entre le CECR et ce référentiel est le fait que la grille canadienne ne s'interdise pas les formulations négatives. Aux niveaux A et $\mathrm{B}$, elles sont plutôt attendues. Un candidat de niveau $B$ en interaction orale, par exemple, pourra faire des fautes de grammaire et de prononciation ( $«$ Bien qu'il puisse y avoir de nombreuses erreurs et lacunes sur le plan de la grammaire, de la prononciation, du vocabulaire et du débit, la communication ne s'en trouve pas sérieusement entravée. Toutefois, on ne doit pas s'attendre à ce qu'un candidat de ce niveau puisse faire face à des situations délicates, ou à des situations où l'on discute de questions abstraites ou subtiles, ou encore, à des situations où l'on formule des hypothèses et où l'on défend des arguments »). Là où les formulations sont plus surprenantes en regard du Cadre, c'est au niveau C, clairement défini comme différent du niveau de langue d'un natif :

[...] il ne faut pas s'attendre à ce qu'elle [la personne] le fasse avec la même aisance et au même rythme qu'un locuteur natif. Il peut y avoir des lacunes sur le plan de la prononciation, de la grammaire et du vocabulaire, mais celles-ci n'entravent pas sérieusement la communication.

Le niveau C n'est pas celui du locuteur natif mais de la personne capable d'accomplir une série de tâches aussi bien qu'un natif. Sur cette distinction encore, le système canadien se montre plus précis que le Cadre. En effet, le niveau C2 du Cadre n'est pas nécessairement celui du locuteur natif, mais d'une personne capable d'accomplir en langue étrangère des tâches complexes sur le plan cognitif.

La prise en compte de la langue de spécialité constitue la troisième particularité du système canadien. La CFP-PSC est même allée jusqu'à décrire les compétences particulières et les a ventilées en deux catégories. La première, désignée par la lettre $\mathrm{P}$, renvoie d'une part à l'expertise dans une des deux langues officielles (la langue comme objet de l'expertise), d'autre part à l'expertise professionnelle en l'une des deux langues officielles (la langue comme medium de l'expertise). ${ }^{16} \mathrm{En}$ pratique, $\mathrm{P}$ fait référence aux compétences en matière de langue spécialisée ou de spécialité et distingue clairement langue générale, parlée par tout natif, et langue spécialisée dans un type de discours, soit du praticien de la langue, soit de l'expert du domaine. Ainsi, un locuteur natif peut très bien ne pas être « $\mathrm{P}$ ", alors que peut l'être un non natif connaissant la langue d'une spécialité particulière. $\mathrm{P}$ renvoie aux langues de spécialité dans le sens plus large de "langue des usages sociaux spécialisés", puisque la culture est sociologiquement similaire dans les deux langues officielles et que le bilinguisme considéré par l'administration canadienne concerne les seuls français et anglais tels que pratiqués au Canada. 


\subsection{Validité du système}

\subsubsection{Validité du contenu} accomplir dans le poste. Un exemple de cette liste pour les postes de chef de la validation et des recouvrements, commis de bureau, méthodologiste, rédacteur-réviseur et secrétaire est fourni par la CFP-PFC (CFP-PFC 2006e). On peut ainsi dire que le contenu de l'évaluation est valide.

\subsubsection{Validité simultanée des critères d'évaluation}

Le gouvernement canadien, conscient de l'importance d'une évaluation fiable, tient à la disposition des jurys des exemples de performances sur support vidéo, disponibles pour les deux langues officielles, pour illustrer les niveaux A, B et C en situation d'interaction orale.

\subsubsection{Validité prédictive}

La CFP-PSC tient compte de l'évolution linguistique des candidats à un poste par plusieurs moyens. D'abord, le test de langue prévoit la possibilité, pour un candidat dont le niveau est supérieur à $C$ dans une compétence donnée, d'être exempté d'autres épreuves pour les compétences que le jury juge permanentes et qui ne seront pas testées par la suite. Cependant, le test envisage aussi la déperdition des compétences langagières. Lorsque le jury pense que le niveau du candidat risque de baisser pour une compétence donnée, il remplit une case spéciale indiquant que le niveau devra être maintenu. La question se pose de savoir sur quels critères le jury décide qu'un candidat risque de perdre ses compétences, ou au contraire ce qui lui fait penser que de telles compétences sont permanentes. Par ailleurs, on peut se demander quelle est la force prescriptive de la décision du jury. Le candidat peut-il, par exemple, décider de repasser l'épreuve de langue s'il pense qu'il a amélioré son niveau?

\subsubsection{Validité théorique}

C'est le Conseil du Trésor (Secrétariat du Conseil du Trésor du Canada, 2006) qui détermine dans sa politique quels postes doivent être « bilingues ». Pour l'administration canadienne (CFP-PFC 1993), «bilingue » ne signifie pas possédant le même niveau, en principe très élevé, dans deux langues, ni ayant deux langues maternelles, mais seulement capable de fonctionner en français et en anglais ${ }^{17}$ avec au moins le niveau B. Un locuteur au niveau A n'est pas considéré comme bilingue. Il l'est au niveau C, même s'il commet fautes et erreurs.

Le bilinguisme dont il est question dans le contexte canadien concerne la capacité à fonctionner en français et en anglais, mais pas dans d'autres langues, de sorte que le ministère de la Justice (ministère de la Justice du Canada, 2003) considère qu'une personne maitrisant plusieurs langues, mais pas l'une des deux langues officielles, est tout de même considérée comme unilingue. Ce sont directement les besoins des administrés dans l'une des deux langues officielles qui commandent le recrutement à des postes dans lesquels est imposée la maîtrise à la fois du français et de l'anglais. Pourtant, 
si des besoins se font jour dans d'autres langues (en particulier au contact des populations indiennes, inuit et immigrées), ils ne sont pas pris en compte par le Conseil du Trésor. Le nouveau Décret d'exemption concernant les langues officielles dans la fonction publique (Gouvernement du Canada 2005) a même pour objectif de "favoriser l'accès des Canadiens et des Canadiennes unilingues aux postes bilingues de la fonction publique fédérale ", mais pas de refléter le multilinguisme, et pas seulement le bilinguisme anglais / français, des administrés.

41 Une fois déterminés les besoins en postes «bilingues», on effectue une analyse des besoins langagiers dans ces postes. L'analyse de la CFP-PFC (CFP-PFC 2005) tient compte des compétences mises en œuvre dans le poste et de celles qui ne le sont pas. En pratique, la détermination des compétences dans chaque langue se fait en deux étapes: 1) énumération des tâches à réaliser et des langues dans lesquelles elle doivent être réalisées ; 2) indication du niveau de compétence dans la ou les langues concernées.

Enfin, les candidats à tous les corps sont invités à se soumettre à des tests de langue qui permettront d'établir leur "profil linguistique ». Les candidats recrutés à des postes bilingues devront apporter la preuve de leur compétence en langue au niveau requis par le poste.

Le système d'évaluation en langue en vigueur dans l'administration canadienne semble donc théoriquement valide; le fait de ne tenir compte des besoins que dans les deux langues officielles relève évidemment d'un choix politique.

\subsubsection{Validité de la notation}

Les services qui établissent le profil linguistique des postes bilingues reçoivent des instructions précises du CFP-PSC. Les jurys disposent du référentiel, valable pour tous les postes bilingues et, pour l'oral, reçoivent des instructions précises sur le déroulement de l'épreuve et le contenu des questions. Les épreuves orales sont enregistrées, officiellement dans un but d'archivage, dans un dossier linguistique propre à chaque candidat. Ces derniers ont accès aux référentiels. Le fort cadrage des jurys et la relative transparence des épreuves garantissent la validité de la notation.

\subsubsection{Validité apparente}

L'examen de langue nous semble effectivement valide. Le descriptif des compétences est précis et prévoit les deux extrémités de la compétence, l'absence de compétences et la compétence maximale, laquelle est déclinée de plusieurs manières. Les candidats sont informés des critères d'évaluation, des sujets sur lesquels portera la conversation à l'oral, de la confidentialité de leurs réponses, de l'archivage de leur prestation et bénéficient en outre de conseils pratiques sur la préparation et le déroulement des épreuves. À l'écrit, des exemples de questions sont disponibles avec les réponses sur le site de la Commission de la Fonction Publique. L'administration se donne aussi la peine de répondre aux perceptions des candidats sur le caractère arbitraire et injuste de l'évaluation à l'oral (CFP-PFC 2006f). À l'issue des épreuves, les candidats reçoivent également une rétroaction sur leur performance avec des conseils sur la façon de l'améliorer (CFP-PFC 2006g).Stylistiquement, toujours dans un souci de clarté, les informations à l'intention des candidats sont rédigées à la deuxième personne du pluriel et à l'impératif. ${ }^{18}$ 


\section{Conclusion} l'administration et pour les candidats aux concours. Pour la première, il est crucial de s'assurer que les fonctionnaires exerçant des postes à responsabilité possèdent bien les compétences requises. Les seconds ont besoin de savoir, pour réussir un concours qui se prépare parfois sur plusieurs années, quelles compétences ils doivent maitriser et à quel niveau. En dépit d'enjeux personnels et sociétaux majeurs, l'évaluation en langues aux concours, en France, manque de validité. Plusieurs raisons peuvent expliquer ce phénomène. Il existe d'abord une tension entre deux conceptions de la fonction des concours. Selon une première conception que nous pourrions qualifier d'humaniste, le concours sélectionne sur des critères académiques les candidats dont la culture économique, générale, administrative et souvent linguistique (puisque ce sont les épreuves qui reviennent le plus fréquemment dans les concours), doit être la plus vaste possible. L'autre vision des concours, utilitariste, conçoit le concours comme moyen de sélectionner sur critères professionnels les candidats les plus aptes à remplir les fonctions qu'ils exerceront dans leur métier.

Ces deux conceptions se trouvent devant des difficultés pratiques. La conception humaniste, qui a décidé du choix de l'épreuve de version, considère qu'il existe un absolu de la compétence en langue qui peut s'évaluer au contact d'un texte général. Le candidat compétent doit être capable de percevoir, et si possible de rendre en français, les effets de style du locuteur. Il doit en outre pouvoir dégager de ce texte le sens, la problématique, les enjeux, au besoin grâce à la maïeutique de l'épreuve orale. Cette conception du concours n'intègre pas les recherches concernant l'apprentissage des langues: l'expression en français (en supposant que c'est bien là la L1 du candidat) n'est pas un reflet valide de la compréhension d'un texte. La traduction fait aussi l'impasse sur les langues de spécialité. ceux qui aspirent à des postes où sont requises des compétences dans la seconde langue officielle sont bien en mesure d'effectuer les tâches que leur impose leur fonction. Ce faisant, le système ne vise pas à reconnaître la diversité linguistique de sa population (et ne le permet pas), ni à promouvoir d'autres minorités linguistiques autres que les francophones (qui sont une majorité au Québec). Il s'agit d'un choix politique éloigné du choix français. Ce système entérine la domination de fait de l'anglais dans le contexte nord-américain, tout en reconnaissant les mêmes droits linguistiques aux francophones.

Les mêmes dispositions ne sont, bien entendu, pas possibles en France parce qu'elles seraient inadaptées au contexte européen. Cependant, la fonction publique française semble bien s'être engagée sur la voie utilitariste. Elle a entamé une démarche « métiers » qui consiste à définir les métiers de la fonction publique (243 ont été dénombrés dans la seule fonction publique territoriale) et les compétences qui sont nécessaires. Toutefois, le Répertoire Interministériel des Métiers de l'État ne comporte pas encore d'espace relatif aux langues à maîtriser, par métiers, en fonction du CECR. Peut-être la connaissance de langues étrangères n'est-elle simplement pas indispensable pour l'exercice de ces métiers. Pourtant, le ministère de la Fonction publique (Direction générale de l'administration et de la fonction publique 2005), évoquant la notion d'«espace administratif européen ", entend développer les échanges entre les fonctions publiques 
des pays de l'UE, ce qui suppose des compétences langagières encore non évaluées. D'autres besoins se sont faits jour, notamment la traduction de sites Internet du français vers l'anglais, préconisée par le rapport Bloche (Bloche 1998).

Néanmoins, la démarche "métiers » comporte elle-même des limites. Elle ne permet pas de promouvoir certaines minorités, ni d'assurer leur représentation, ni non plus d'encourager la maitrise de langues rares. La fonction publique territoriale, quant à elle, de création plus récente, combine souvent, pour le recrutement, épreuves théoriques de concours et recrutement à un poste donné après examen d'un curriculum et après entretien.

Dans l'attente d'un choix politique éclairé et plus affirmé, les administrations en France ont intégré des professionnels dans les jurys des épreuves de langues, proposant des formations postérieures au recrutement, toujours dans un choix de langues réduit, et sans résoudre la question de l'hétérogénéité des langues de leurs administrés. La plupart des administrations se trouvent cependant toujours devant une aporie, devant sélectionner des candidats pour un corps (donc pouvant remplir des missions hétérogènes en métropole comme dans les DOM-TOM), non pour un poste. Les épreuves de langues, lorsque les responsables des concours cherchent à les réformer, restent néanmoins perçues comme secondaires par rapport à d'autres épreuves; cette faible importance se reflète dans leur caractère souvent optionnel, voire facultatif et dans les coefficients. Les enseignants de langues, qui constituent l'essentiel des jurys de langues aux concours, se sentent d'autant moins impliqués dans la conception des épreuves de concours, sans doute parce qu'eux-mêmes sont souvent issus d'un recrutement fondé sur le modèle humaniste, bien que les concours d'enseignement comportent un volet pratique ou professionnel. La docimologie les intéresse lorsqu'elle porte sur les concours d'enseignement, qui suscitent des débats passionnés entre membres des sociétés savantes, mais pas lorsqu'elle porte sur les concours administratifs. La minorité qui possède une culture des langues de spécialité est impuissante à conduire une analyse des besoins dans des postes dont elle ignore tout et reste peu, voire pas du tout impliquée dans la formation post-concours, préalable à la prise de poste.

Quelle que soit la formule retenue - maintien d'un statu quo avec une évaluation non valide, chronophage, redondante lorsque les candidats passent plusieurs concours, et donc coûteuse pour les aspirants au statut de fonctionnaire comme pour l'administration, ou bien évaluation des besoins dans les métiers à défaut de pouvoir les évaluer dans les corps et dans les postes - deux mesures demeurent souhaitables. D'une part, les compétences langagières devraient être évaluées de façon distincte sans passer par le français, ce qui implique la fin des épreuves de version et thème. D'autre part, pour les concours où les langues étrangères revêtent un caractère obligatoire, il pourrait être demandé un niveau minimal au candidat dans chacune des cinq compétences définies par le Conseil de l'Europe (lire, écrire, parler, comprendre et interagir). La charge de la preuve du niveau pourrait revenir au candidat, qui devrait produire un Portfolio Européen des Langues (PEL) dans le Dossier duquel figureraient les attestations de niveau nécessaires, délivrées par des autorités accréditées. Une évaluation unique valable pour tous les concours où sont requises des compétences en langues, à l'image de celle pratiquée au Canada, permettrait d'accroître la transparence des critères d'évaluation. Le «profil linguistique » serait établi pour toutes les langues que maitrise le candidat, soit en le testant selon les recommandations du Conseil de l'Europe en utilisant la grille développée par l'équipe de chercheurs de J.C. Alderson (Alderson et al. 2006), soit en 
examinant son PEL ou ePEL, pour ensuite affecter le lauréat au plus près des compétences en langues identifiées.

L'observation des modalités de recrutement en langues dans au Canada se révèle un champ particulièrement riche pour les spécialistes d'anglistique, civilisationnistes et docimologues, en particulier pour contribuer à la diffusion des bonnes pratiques d'évaluation et, au-delà, de management public. Cette étude nous conduit à établir également d'autres observations. Le nouveau référentiel canadien se montre plus précis dans certaines de ses formulations que le tableau des niveaux du Cadre, dont les imprécisions ont été soulignées par Alderson (Alderson et al. 2006). Le système canadien propose également une définition de la maîtrise de la langue spécialisée. Une comparaison des modalités d'évaluation en œuvre dans les fonctions publiques d'autres pays anglophones permettra d'élargir cette étude en comparant la mise en œuvre du CECR et d'autres référentiels, puisque les opérateurs dans le domaine de l'évaluation de la fonction publique dans et hors Europe tendent à développer leurs propres outils.

\section{BIBLIOGRAPHIE}

Alderson, J. C., N. Figueiras, H. Kuijper, G. Nold, S. Takala et C. Tardieu. 2006. « Analysing Tests of Reading and Listening in Relation to the Common European Framework of Reference: The Experience of the Dutch CEFR Construct Project ». Language Assessment Quarterly 3/1, 3-30.

Bloche, Patrick. 1998. Le Désir de France, la présence internationale de la France et la francophonie dans la société de l'information, Rapport au Premier ministre, La Documentation française. 12/12/07 < http://lesrapports.ladocumentationfrancaise .fr/cgi-bin/brp/telestats.cgi? brp_ref $=994000906 \&$ brp_file=0000.pdf $>$.

CFP-PSC. 1993. L'évaluation des compétences. L'établissement du profil linguistique pour les postes bilingues.

CFP-PSC. 2003. Online tests en ligne. Mise en cuvre de la phase II : rapport aux partenaires. Commission de la fonction publique du Canada. 12/12/07, <http://www.psc-cfp.gc.ca/ppc/ phase2_report_f.pdf>.

CFP-PSC. 2005. Normes de qualification relatives aux langues officielles. Modernisation de la gestion des ressources humaines. 12/12/07 <http://www.psagency-agencefp.gc.ca/hrmm-mgrh/ Qualstandard/section03_f. asp>.

CFP-PSC. 2006a. Évaluation de la langue seconde : test d'interaction orale. Commission de la fonction publique du Canada. 12/12/07 <http://www.psc-cfp.gc.ca/ppc/sle_pg_01c_f.htm>.

CFP-PSC. 2006b. Évaluation de la langue seconde : test de compréhension de l'écrit. Commission de la fonction publique du Canada. 12/12/07 <http://www.psc-cfp.gc.ca/ppc/sle_pg_02_f.htm>.

CFP-PSC. 2006c. Évaluation de la langue seconde : test d'expression écrite. Commission de la fonction publique du Canada. 12/12/07 <http://www.psc-cfp.gc.ca/ppc/sle_pg_03_f.htm>. 
CFP-PSC. 2006d. Le nouveau Décret d'exemption concernant les langues officielles dans la fonction publique. Commission de la fonction publique du Canada. 12/12/07 <http://www.psc-cfp.gc.ca/ centres/ol/eao/eao_f.htm>.

CFP-PSC. 2006e. Établissement du profil linguistique pour les postes bilingues. Commission de la fonction publique du Canada. 12/12/07 <http://www.psc-cfp.gc.ca/ppc/sle_pg_06_f.htm>.

CFP-PSC. 2006f. Test d'interaction orale : perceptions (erronées) et faits. 12/12/07 <http://www.psccfp.gc.ca/ppc/sle_oi_test_f.htm>.

CFP-PSC. 2006g. Évaluation de la langue seconde. Test d'interaction orale. Rétroaction au candidat ou à la candidate. 12/12/07 <http://www.psc-cfp.gc.ca/ppc/sle_feedback_f.htm>.

Gouvernement du Canada. 2005. « Décret d'exemption concernant les langues officielles dans la fonction publique ». Gazette du Canada. 12/12/07 <http://canadagazette.gc.ca/ partII/2005/20051214/html/si118-f.html>.

Direction générale de l'administration et de la fonction publique. 2005. «Échanges entre les fonctions publiques des pays de l'UE ».12/12/07 <http://www.fonction-publique.gouv.fr/ article392.html>.

Division des langues vivantes, Strasbourg. 2000. Apprentissage des langues et citoyenneté européenne, Un cadre européen commun de référence pour les langues : Apprendre, enseigner, évaluer. 12/12/07 < http://www.coe.int/T/DG4/Portfolio/documents/cadrecommun.pdf>.

Division des langues vivantes, Strasbourg. 2003. Relier les examens de langues au Cadre européen commun de référence pour les langues : Apprendre, enseigner, évaluer (CECR). Manuel avant-projet. 12/12/07 <http://www.coe.int/T/DG4/Portfolio?L=F\&M=/documents_intro/Manuel.html>.

Hughes, Arthur. [1988] 2003. Testing for Language Teachers. Cambridge : Cambridge University Press.

Ministère de l'Emploi et de la fonction publique. 2007. « Avis de concours et de vacance d'emploi », Journal Officiel, 12 mars 2007, <http://www.fonction-publique.gouv.fr/IMG/ calendrier_prev_20070317.pdf>.

Ministère de la Justice du Canada. 1985. Lois sur les langues officielles. Lois et règlements codifiés. 12/12/07 <http://lois.justice.gc.ca/fr/showtdm/cs/O-3.01>.

Ministère de la Justice du Canada. 2003. Loi sur l'emploi dans la fonction publique, Lois et règlements codifiés. 12/12/07. <http://lois.justice.gc.ca/fr/showtdm/cs/P-33.01>.

Méthy, A.-M. et B. Monglon. 2000a. L'anglais des concours administratifs. Paris : Ophrys.

Méthy, A.-M. et B. Monglon. 2000b. Le Secret dans les concours administratifs. Communication au colloque du C.E.R.L.A.C., Université Panthéon-Sorbonne.

Ministère de l'Éducation nationale, inspection générale de l'éducation nationale. 2005. Pilotage et cohérence de la carte des langues, La Documentation française. 12/12/07 < http:// lesrapports.ladocumentationfrancaise.fr/cgi-bin/brp/telestats.cgi? brp_ref $=054000373 \&$ brp_file=0000.pdf $>$.

Ministère de la Fonction publique, Observatoire de l'emploi public. 2005. Rapport annuel 2004-2005. 12/12/07 <http://www.fonction-publique.gouv.fr/IMG/Oep-rapport2004-2005.pdf>.

Ministère de la Fonction publique, Direction générale de l'administration et de la fonction publique. 2006. Répertoire interministériel des métiers de l'Etat $1^{e}$ édition 12/12/07 <http:// www.fonction-publique.gouv.fr/IMG/RIME_version_16062006.pdf>. 
Ministère de la Justice du Canada. 2005. Règlement sur les langues officielles-communications avec le public et prestation des services. Lois et règlements codifiés. 12/12/07 <http://canadagazette.gc.ca/ partII/2007/20070822/html /sor172-f.html>.

Petit, Michel. 2006. «Les descripteurs du Cadre : quelle conception de la langue de spécialité ?» Journées de l'EA2025 BORDEAUX 2 - IUFM d'Aquitaine Le Cadre européen commun de référence pour les langues : enjeux théoriques et implications pratiques, Bordeaux, 2-3 février 2006. 12/12/07. < http://www.langues-vivantes.u-bordeaux2.fr/pagesperso/michelpetit/pdf/06BxCadreDescr2.pdf $>$.

Puren, Christian. 1988. Histoire des méthodologies de l'enseignement des langues. Paris : Nathan.

Secrétariat du Conseil du Trésor du Canada. 2006. À notre sujet. 12/12/07. <http://www.tbssct.gc.ca/common/us-nous_f.asp\#CT>.

Tasca, Catherine. 2003. Rapport sur l'usage de la langue française et les pratiques linguistiques dans les entreprises. Ministère de la Culture et de la communication. 12/12/07. <http:// www.culture.gouv.fr/culture/dglf/rapport/2003/Rapport_au_parlement_2003.pdf>.

\section{NOTES}

1. Loi sur les langues officielles (LLO) de 1985 , loi sur l'emploi dans la fonction publique (LEFP) de 2003, Décret d'exemption concernant les langues officielles dans la fonction publique (DELOFP) de 2005.

2. L'épreuve d'anglais du concours de lieutenant de police, session 2003, Gif-sur-Yvette.

3. Par exemple, l'épreuve d'anglais de technicien des parcs nationaux est seulement qualifiée d'épreuve écrite.

4. Toutefois, les renseignements sur les épreuves de concours sur les sites-toile des administrations étaient parfois indiqués avant et peu de temps après le concours, puis étaient retirés. Les mises à jour de la base de données s'effectuant tous les ans au printemps, il est possible que des informations nous aient échappé.

5. Elle est présentée sous forme de tableau (voir annexe 1).

6. Tel est le cas, par exemple, du concours d'architecte urbaniste de l'État.

7. Les profils des postes de chargé de recherches pour le Ministère de l'équipement indiquent qu'une bonne connaissance de l'anglais est indispensable, alors que le concours ne prévoit pas d'épreuve de langue <http://www2.equipement.gouv.fr/recrutement/presentations_generales/ CRDR-06-pg.pdf>.

8. <http://www.education.gouv.fr/bo/2003/30/MENE0301542N.htm >.

9. Le concours d'officier de port est le seul à renvoyer à un corpus, le vocabulaire normalisé des affaires maritimes.

10. Le plus souvent, les concours de catégorie A sont ouverts aux titulaires d'un diplôme à niveau bac +3 , les concours de catégorie $B$ aux titulaires du bac et les concours de catégorie $C$ aux titulaires du Brevet des collèges. Dans la pratique, les conditions d'inscription varient selon l'administration organisatrice et on observe que certaines administrations relèvent le niveau de diplômes requis pour passer certains concours de catégorie $\mathrm{B}$, de sorte que le diplôme à $\mathrm{Bac}+3$ est demandé pour pouvoir concourir à un poste de catégorie $\mathrm{B}$ là où il permet de passer les concours de catégorie A d'autres ministères. Par exemple, le concours de lieutenant de police (B) recrutait à $\mathrm{Bac}+3$ à la session 2003. En pratique, la plus grande partie des lauréats de ce concours possède le niveau Bac +5 . 
11. Le jury du concours de lieutenant de police par exemple n'utilise pas de référentiel pour juger de la difficulté du texte. Un jury composé de professionnels se réunit et sélectionne des textes dont la difficulté leur semble correspondre au niveau du baccalauréat.

12. Ou validité hypothético-déductive, ou validité conceptuelle, ou de construct, de construit, de construction.

13. Ou validité perçue, ou validité faciale.

14. À l'exception du concours d'officier de port et de certains concours du ministère des Affaires étrangères.

15. Les italiques sont de notre fait.

16. «La cote P est utilisée dans le profil linguistique pour deux types de compétences linguistiques spécifiques. Ce sont, d'une part, les compétences reliées à la langue, normalement acquises au moyen d'une formation spécialisée (y compris l'audiotypie ; la relecture de textes ; la rédaction, la révision ou la correction de textes ; la traduction ; l'interprétation); d'autre part, la compétence de spécialiste ou d'expert dans une langue officielle ou les deux (par exemple, celle utilisée pour enseigner l'anglais ou le français) ».

17. «Ces tests et ces normes s'imposent pour tous les groupes professionnels (...). Les postes qui sont désignés bilingues en vertu de la politique du Conseil du Trésor, parce qu'ils consistent à offrir des services au public ou à assumer des fonctions de surveillance, doivent être au moins au niveau B pour chaque compétence. Toutefois, pour les postes des catégories du soutien administratif ou de l'exploitation, le niveau A peut être indiqué si les fonctions et les responsabilités du poste le justifient » (p. 2).

18. L'ensemble des différences décrites ci-dessus entre l'évaluation en langue à l'entrée de l'administration en France et au Canada est décrit en annexe 3.

\section{RÉSUMÉS}

Cet article se propose de comparer les modalités de l'épreuve de langue aux concours d'accès à la fonction publique en France et au Canada. Concernant la France, l'étude concerne les concours des fonctions publiques d'État et territoriale. Une typologie des épreuves laisse apparaitre un nombre restreint de types d'épreuves, dominées, à l'écrit comme à l'oral, par la version, accompagnée voire remplacée à l'oral par une conversation. Ceci reflète une organisation des épreuves extrêmement décentralisée. Les besoins en langues sont rarement envisagés par poste ; ils le sont plutôt par corps. Au Canada, l'évaluation du niveau de compétence dans la seconde langue officielle vient d'être revue. Les candidats sont évalués en fonction d'un référentiel qui pallie certains points problématiques du référentiel établi par le Conseil de l'Europe, le Cadre européen commun de référence (CECR), comme, par exemple, la place des langues de spécialité. Les besoins en langues par postes sont analysés grâce à ce référentiel. Nous proposons enfin quelques pistes pour améliorer la validité des épreuves de langues aux concours administratifs en France.

Language testing in competitive entrance exams for the French civil service is compared to the equivalent in Canada for entrance to the Canadian civil service. On the French side, this covers national, regional and local administrative posts. Analysis reveals a limited range of language test types, whether written or oral, confined primarily to translation from English into French and/or conversational exchange. The absence of any centralised form of test organisation is also 
apparent: tests vary according to ministries and administrations, and language needs are hardly ever defined according to function or job type. Canada has recently reviewed its official second language assessment procedures. Candidates are to be assessed henceforth by means of a grid which overcomes a number of problem areas identified in the CEFR such as, for example, languages for specific purposes. In both French and English, language needs are analysed with this new grid in terms of function or job type. Suggestions are made to better validate language tests in French civil service entrance exams.

\section{INDEX}

Keywords : assessment, CEFR, civil service entrance examination in Canada, civil service entrance examination in France, Common European Framework of Reference, language for specific purposes, language policy

Mots-clés : Cadre européen commun de référence pour les langues, CECRL, concours administratif au Canada, concours administratif en France, évaluation, langue de spécialité, politique linguistique

\section{AUTEUR}

\section{ANNE-MARIE BARRAULT-MÉTHY}

Anne-Marie Barrault-Méthy est maître de conférences à l'Université Montesquieu-Bordeaux 4 et membre de l'Équipe d'accueil 4140 Laboratoire Culture, Éducation, Sociétés (LACES). Ses recherches portent sur les politiques linguistiques en relation avec le Cadre européen commun de référence du Conseil de l'Europe. Elle co-coordonne la création et l'utilisation d'un ePEL dans son université avec des universités d'Europe partenaires du projet. Elle assure également la préparation à l'épreuve d'anglais aux concours administratifs de catégorie A et B à l'IPAG de Poitiers. ammethy@u-bordeaux4.fr 